ORIGINAL ARTICLE

PRACA ORYGINALNA

\title{
IMMUNOHISTOCHEMICAL AND GENETIC PROGNOSTIC FACTORS OF NEOADJUVANT CHEMORADIOTHERAPY EFFICACY IN PERSONALIZED TREATMENT OF LOCAL ADVANCED RECTAL CANCER
}

DOI: 10.36740/WLek202003136

\author{
Vitalii V. Zvirych, Yuriy I. Michailovich, Oleksandr I. Gorbach, Natalia M. Khranovska \\ NATIONAL CANCER INSTITUTE, KYIV, UKRAINE
}

\begin{abstract}
The aim: The aim of our study was to define the factors that can robustly predict a response to neoadjuvant chemoradiotherapy (NCRT) in patients with local advanced rectal cancer (LARC) and prognosis factors of progression free survival (PFS) using molecular (8-oxodGu), immunohystochemical (Ki-67) and genetic (GSTP1 and MTHFR genes polymorphism) markers.

Materials and methods: GSTP1 and MTHFR polymorphisms were studied by real-time PCR on tumour material from 110 patients with LARC. Ki-67 protein expression was assessed using rabbit monoclonal antibodies to Ki-67 (Dako, Denmark) on EnVision ${ }^{T M}$ FLEX detection system (Dako, Denmark). 8-oxodGu level in eluate was measured by spectrophotometry. Results: Patients from both groups showed significant pathomorphological response to NCRT. It is robust correlation between 8-oxodGu levels in patients' blood and their response to CRT (mrTRG scale) in MG was determined. Oxaliplatin-containing chemotherapy promotes statistically significant decrease of 8-oxodGu levels. With the decrease of Ki-67 protein expression level the probability of tumour relapse increases. It is determined that critical value of Ki-67 protein expression level makes less than 27 and tumour relapse probability in this case makes 50\%. Tumour relapse risk in patients with GSTP1 and MTHFR polymorphism is 12.3 and 16.3 times higher than in patients who do not carry such polymorphism, respectively. Combination of GSTP1, MTHFR polymorphisms and Ki-67 protein expression factors determines prognostic probability of tumour relapse within 51-99\%.

Conclusions: 8-oxodGu level can serve as independent prognostic factor of NCRT efficacy in patients with LARC. Combination of GSTP1, MTHFR genes polymorphism with Ki-67 protein expression decrease enables monitoring and robust prognosis of LARC relapse.
\end{abstract}

KEY WORDS: neoadjuvant chemoradiotherapy, rectal cancer, prognosis factors.

Wiad Lek. 2020;73(3):589-596

\section{INTRODUCTION}

Modern tactics for localized rectal cancer treatment is strictly standardized and regulated by International Guidelines (NCCN Guidelines v.2. 2017) [1]. In contrast to localized forms, for LARC that features high probability of relapse and inner organs systemic lesion there still is no finally formulated treatment and diagnostic standard and until very recently even definition by itself was not formulated [2].

To reach proper resectability levels, NCRT is the only solution it was supported by $100 \%$ of the experts and certified as the form of Consensus (2013). This procedure enables "stage downgrade" - transformation of locally spread form into localized one, significantly improves postponed treatment results and decreases the number of local relapses. Tumour regression degree after NCRT directly correlates to survival rates and local relapse frequency [3].

Tumour reaction on CRT can vary from complete pathological regression (pCR) to full resistance (according to different authors, regression value makes from 3 to $54 \%$ ) [4]. Tumour regression degree after NCRT directly correlates to survival rates and local relapses frequency. Based on randomized clinical trials, it is proved that preliminary course of radiotherapy both fluorouracil and with oxaliplatin promotes tumour regression. Such effect enables definitive organ preserving surgery and leads to local relapses decrease in postsurgical period. NCRT is integral part of combined LARC treatment and can induct partial, significant and total regression of primary tumour with long lasting PFS $[5,6]$.

Nowadays, the main goal of LARC NCRT is to decrease the relapse development risk in true pelvis by means of maximal reduction of primary tumour size and tumour process stage downgrade. To meet this goal, modified regime of neoadjuvant chemotherapy (NCT) - replacement of fluoropyrimidine monochemotherapy to oxaliplatin polyhemotherapy, looks as a very promising strategy. Yet, growing NCRT interruption frequency resulting from toxicity increase remains to be an issue. Positive impact on general ten-years survival was showed only in one of four multi-institutional randomized research published as for today [7-10]. Nevertheless, in recently published scientific 
reports there is no any data about any of chemotherapy agents' advantages. The results of STAR-01, ACCORD 12/0405-Prodige-2, NSAPB P-04, PETACC-6 randomized studies did not demonstrate significant improvement in pathological complete locoregional response and increase in survival rates connected to oxaliplatin addition to the treatment regimen. Moreover, in patients treated with oxaliplatin toxicity increase of 3-4 grade was observed [11-33].

Opposed to this, German CAO/ARO/AIO-04 studies involving integration of oxaliplatin into fluoropyrimidine-based NRT of rectal cancer demonstrated higher pathological complete response ( $\mathrm{pCR}-17 \%$ versus $13 \%$; $\mathrm{p}=0.038)$, higher 3 -years PFS (75.9\% versus $71.2 \%$; $\mathrm{p}=$ 0.03 ) and no increase of general toxicity in oxaliplatin group $[14,15]$.

Though, there is no clear data about efficacy of NCRT with multimodal oxaliplatin including treatment in patients with LARC. According to ESMO and NICE recommendations, improved treatment results can be expected in field of personalized approach that considers immediate and postponed relapse risks. The problem of choosing right NCRT should be further investigated taking into account clinical, radiological, pathomorphological data and prognostic markers.

Other very important and insufficiently studied aspects are immunohistochemical and genetic peculiarities of tumour as prognostic factor in RC.

Level of malignancy maturation depends on intensity of tissue and cellular atypism and mitotic activity level. To assess cell proliferation in tumor, series of markers are commonly used. Conventional cell proliferation marker Ki-67 is one of them. This protein is expressed in proliferating cells during G1 phase and is absent in G0 resting cells [16]. This makes Ki-67 clinically significant proliferation marker for prognosis of several cancer types [17-21].

Clinical trials of Z. Pap et al. showed that in adenoma of the large intestine the Ki-67 expression level directly correlates with seriousness of dysplastic changes [22]. Several studies showed that significant Ki-67 expression is mainly connected to lower overal survival rates [23-27], while other studies highlight that high Ki-67 expression correlates with improvement of general survival and better oncological prognosis [28-30].

It is well known that gene polymorphisms participate in colorectal cancer pathogenesis and many chemotherapeutical drugs metabolism. Individual peculiarities of glutathione S-transferase (GSTP1) and methylene-tetra-hydro-folate-reductase (MTHFR) enzymatic activity mediated by gene polymorphism can predict rectal cancer and development of the resistance to NCT (oxaliplatin, 5-fluorouracil, irinotecan). GSTP1 and MTHFR polymorphism detection in patients with $\mathrm{RC}$ can be efficient for predicting tumor cell response to chemotherapy and toxicity effects of NCT [31-33].

It is very important that MTHFR participates in antineoplastic agent metabolism (methotrexat, fluorouracil). 5-fluorouracil acts via fluorodeoxyuridine monophosphate and inhibits thymidilate synthase [34]. Drop of MTHFR fermentative activity leads to increase of methylenetetrahydrofolate level and thereby enhances 5-fluorouracil cytotoxicity. MTHFR gene polymorphism and efficacy of 5-fluorouracil therapy were estimated in experimental research and clinical trials $[35,36]$.

It is known that chemical cancerogens such as polycyclic aromatic hydrocarbons and heterocyclic aromatic amines are connected to RC; aromatic amines and some of cytostatins such as platinum products, anthracyclines and steroid hormones are the substrates for GSTP1 enzyme. Substitution of adenine for guanine in position 313 of the $5^{\text {th }}$ exon in GSTP1 gene leads to isoleucine substitution for valine in position 104 and reduction of its affinity to electrophilic substances [37].

Moreover, polymorphic variants of GSTP1 and MTHFR are the risk factor for gastrointestinal and cardiovasculare toxicity that can be associated with development of resistance to chemotherapy. Genotyping of MTHFR C677T polymorphism enables personalization of chemotherapy agents.

In a number of scientific studies, much attention is paid to tumor microenvironment that is one of the key progression factors of tumor resistance to chemotherapy [38]. In addition, further investigations of superoxide radicals and 8-oxoguanine influence as potential markers of RC development are very promising [39-41].

8-oxodGu present in DNA in the absence of reparation can cause cell cycle arrest and apoptosis. Most scientific research indicate the role of oxidative stress in pathogenesis of colon cancer. Chang et al. report that 8-oxodGu content in blood serum can serve as a sensitive biomarker in patients with rectal adenocarcinoma [42] and is considered as a high-informative marker of tumourogenesis [43] and important marker of tumor response to treatment [44].

As a result, this review of combined LARC treatment based on immunohistochemical and genetic prognosis factors implies many complicated unresolved issues that should be addressed in future scientific studies.

\section{THE AIM}

Our aim was to define prognostic factors of NCRT efficacy in patients with LARC, namely the prognostic factors of PFS using molecular (8-oxodGu), immunohystochemical (Ki-67) and genetic (GSTP1 and MTHFR genes polymorphism) markers.

\section{MATERIAL AND METHODS}

This research is based on the retrospective data analysis from 110 patients, who underwent combined treatment of LARC in Oncocolonoproctology Department of National Cancer Institute from 2016 to 2019 years.

The diagnostic algorithm included estimation of overall patient's status according to ECOG scale, fibrocolonoscopy with biopsy and morphological verification, magnetic resonance imaging (MRI) of thoracic, abdominal and pelvic organs with intravenous contrast, laboratory tests and 
electrocardiography. Local staging was conducted using Philips Intera 1,5 T MRI scanner according to MERCURY protocol [45]. All patients confirmed by MRI to be CRM+ were included into the research. It means tumor invasion, metastatic transformation of lymph nodes or extranodal tumor deposits, tumor infiltration of mesorectal soft tissue to a distance of not less than $1 \mathrm{~cm}$ from the edge. Accuracy of tumor topography in relation to mesorectal fascia played a critical role in choosing treatment plan, namely administration of NCRT and was the most important reference point for total mesorectal excision (TME). Estimation of tumor regression grade according to MRI is based on mrTRG and RECIST 1.1 (Response Evaluation Criteria in Solid Tumours) criteria.

All patients with LARC considering NCRT method were randomized at a ratio of 1:1 with respect to cTNM-pTNM (T3-4 N0-2 M0, CRM+) index.

Patients from MG undewent radiotherapy with total radiation dose of $50.4 \mathrm{~Gy}$ (28 sessions 1.8 Gy each) and polychemotherapy according to CAPOX regimen with oxaliplatin in non-adjuvant regime: capecitabine $200 \mathrm{mg} /$ $\mathrm{m}^{2}$ in two equal doses, one in the morning and one in the evening, peroral in 30 minutes after meals from day 1 to day 14; oxaliplatin $130 \mathrm{mg} / \mathrm{m}^{2}$ intravenous on day one of the cycle. The interval between cycles made 21 days.

Patients from CG underwent radiotherapy with total radiation dose of $50.4 \mathrm{~Gy}$ (28 sessions 1.8 Gy each) and monochemotherapy based on fluoropyrimidines in non-adjuvant regime: capecitabine $200 \mathrm{mg} / \mathrm{m}^{2}$ twice a day. The interval between cycles made 5 days. In general, every patient underwent two cycles of chemotherapy. Toxicity effects of NCRT were evaluated according to CTC-NCIC scale (version 4.03, 2010). Eight days after NCRT course its efficacy in all patients was evaluated using MRI according to the protocol mentioned above.

Eight weeks after NCRT course, all patients underwent TME. Histopathological estimation of TME quality was conducted according to P. Quirke method. Post-surgical complications were estimated according to Clavien-Dindo-Strasberg classification within 1-30 days period starting from the day of surgery. All observations are in compliance with Ethics Cometee requirements.

To study GSTP1 and MTHFR genes polymorphism, genomic DNA was extracted from paraffin embedded tumor material using method of NC adsorption on silica-membrane (PureLink Genomic DNA Kits, Invitrogen, USA). DNA concentration was measured using NanoDrop-1000 spectrophotometer (Thermo Scientific, USA). Prior to amplification, DNA concentration was from 2 to $8 \mathrm{ng} / \mu \mathrm{L}$. GSTP1 and MTHFR genes polymorphisms were studied by allele-specific real-time PCR on 7300/7500 Real-Time PCR Systems (Applied Biosystems, USA). To study A313G, GSTP1 and C677T MTHFR single nucleotide polymorphisms TaqMan Probes of MGB-type were used. TaqMan Probes and primer sequences were selected using Primer Express ${ }^{\oplus}$ Software v3.0 (Applied Biosystems, USA) and synthesized by Applied Biosystems, USA. PCR reaction conditions were as following: $50^{\circ} \mathrm{C}$ for $2 \mathrm{~min}, 95^{\circ} \mathrm{C}$ for $10 \mathrm{~min}$, followed by 45 cycles at $92{ }^{\circ} \mathrm{C}$ for $30 \mathrm{~s}$ and $60^{\circ} \mathrm{C}$ for $1 \mathrm{~min}$.

The levels and accumulation rates of guanine DNA oxidative damage - 8-oxo-dGuo in neutrophilic blood granulocytes of LARC patients were measured 1 day before surgery [46]. For this purpose, $5 \mathrm{~mL}$ of patient's blood from median cubital vein were collected into centrifuge tube with $1 \mathrm{~mL}$ of Trilon B. Neutrophils were separated from according to H.T. Lee [42]. The 8-oxo-dGuo was evaluated in eluate on spectrophotometer.

To study Ki-67 expression, obtained material was fixed in buffered $10 \%$ formaldehyde, $\mathrm{pH} 7.4$, and embedded in paraffin using Histos-5 (Milestone, Italy). Histological slices $5 \mu \mathrm{m}$ in depth were obtained using Microm HM325 (Thermo Scientific, USA). The slices were stained with haemotoxilin and eosin and general tumor estimation was conducted. Immunohistochemistry was performed using rabbit monoclonal antibodies to Ki-67 (Dako, Denmark) on EnVision ${ }^{\mathrm{TM}}$ FLEX detection system (Dako, Denmark). For antigen retrieval, citric buffer with $\mathrm{pH} 6.0$ was used. Primary antibodies were incubated at room temperature for $30 \mathrm{~min}$., secondary antibodies - for $20 \mathrm{~min}$. at room temperature. After this, slices were additionally stained with haemotoxilin Gill. As a positive control, specimens with determined positive reaction were used. The same procedure without primary antibodies served as a negative control. Quantitative microscopic assessment of NCRT efficacy was done according to I. Miller and S. Payene (2003).

Statistical analysis included descriptive statistics (means as $\mathrm{M} \pm \mathrm{m}$ or median $-\mathrm{Me}$, minimal value $-\mathrm{min}$, maximal value - max). Comparison of two independent groups based on one feature was conducted using Mann-Whitney test; comparative assessment of the groups based on variable fraction proportion expressed in nominal or ordinate scale was taken using $\chi^{2}$ Pearson test. Two variables correlation analysis was based on Spearman's rank correlation coefficient. Logistic regression was used for prognostic marker estimation in LARC treatment efficacy. Null-hypothesis of variables equality was rejected when $p<0.05$. Data analysis was carried out in IBM SPSS Statistics 22.

\section{RESULTS AND DISCUSSION}

Fifty seven patients were randomized into MG and fifty three to CG. Average age of the patients in MG makes $59.3 \pm 11.4$ years, in CG $-62.5 \pm 10.2$ years. There were no statistically significant differences in gender, age and anthropometric parameters (body-weight index and total body area) between patients. According to the pathology report, all tumors were adenocarcinomas on varied differentiation stages. Full scale NCRT was conducted in all patients. As a result, the groups were representative.

After the analysis of regression value after NCRT according to MRI (mrTRG and RECIST 1.1 scales), it was determined that tumor response to NCRT was higher in MG - 34 patients (59.7\%) and 37 (64.9\%) than in CG - 25 (47.2\%) and 26 (49.1\%), respectively, however, this difference was statistically insignificant $(\mathrm{p}<0.576 ; \mathrm{p}<0.329)$. 

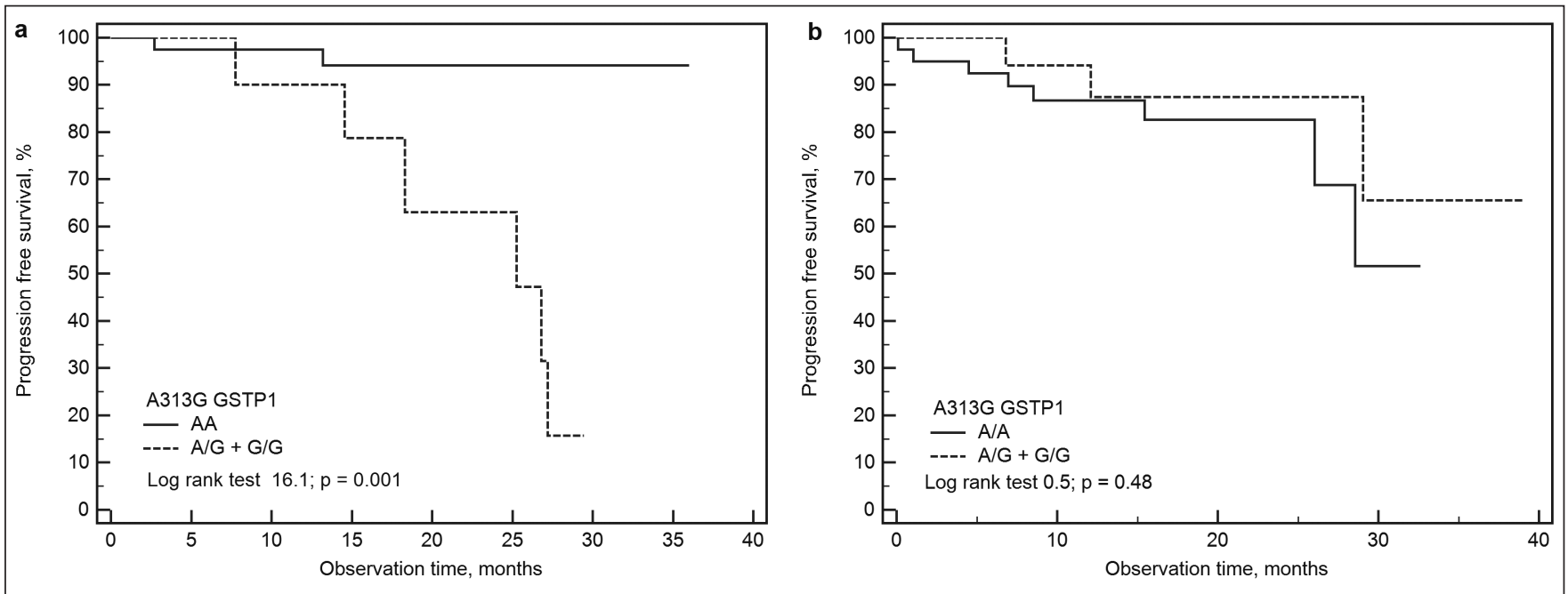

Fig.1. The effect of A313G GSTP1 gene polymorphism on 3-year PFS rate in LARC patients after combined treatment. a - patients received capecitabine as neoadjuvant chemotherapy; $b$ - patients received CAPOX as neoadjuvant chemotherapy.

Significant difference was observed in conversion level of CRM+ to CRM: in MG - 39 (68.4\%) and $24(45.3 \%)$ $(\mathrm{p}<0.05)$.

The estimation of morphometric and topographic anatomy features of tumor regression depending on NCRT scheme showed that in MG the distance between dental line and lower edge of the tumor significantly increased and made $1.1 \pm 0.1 \mathrm{~cm}$ versus $0.7 \pm 0.1 \mathrm{~cm}$ in CG $(\mathrm{p}<0.005)$. The length of the tumor decreased by $28.1 \pm 2.1 \%$ versus $20.4 \pm 2.7 \%(\mathrm{p}=0.024)$, respectively. Thus, positive effect of NCRT on tumor pathogenesis treatment conducted according to the CAPOX scheme and on the quality of TME was proved.

To make oxaliplatin-bazed NCT more personalized, the level of 8-oxodGu in neutrophils from human blood of LARC patients in both groups was measured. Physiological level of 8 -oxo-dGuo makes $0.23 \pm 0.04 \mathrm{nmol} / \mathrm{mLxmin}$ [19].

The statistical analysis of 8-oxodGu level dependent on chemotherapy scheme prescribed, reviled that application of oxaliplatin-bazed NCT as a part of comprehensive treatment of LARC patients aids the significant decrease of studied marker levels: (R2 $=0.465 ; 95 \% \mathrm{CI}: 0.004-$ 0.016, $\mathrm{p}<0.0001)$. Before NCRT 8-oxodGu level in MG was $3.07 \pm 0.08 \mathrm{nmol} / \mathrm{mLxmin}$, in CG $-2.94 \pm 0.06 \mathrm{nmol} /$ mLxmin, after it $-1.96 \pm 0.04 \mathrm{nmol} / \mathrm{mLxmin}$ and $2.72 \pm 0.04 \mathrm{nmol} / \mathrm{mLxmin}(\mathrm{p}<0.001)$, respectively. Thus, we can conclude, that 8 -oxodGu level can be considered as an independent prognostic marker of NCRT efficacy in LARC patients.

Based on logistic regression analysis, the concentration border level of 8-oxodGu in blood is $2.9 \mathrm{nmol} / \mathrm{mLxmin}$. Based on the data supplied above, lowering of 8-oxodGu concentration is recommended before NCRT according to CAPOX scheme when its level is $\geq 2.9 \mathrm{nmol} / \mathrm{mLxmin}$.

All patients in both groups underwent surgery 8 weeks after the NCRT. Surgery extent was determined depending on localization and primary tumor expansion rate. All surgical procedures were R0-resections. In MG 37 patients (65\%) and in CG 26 patients (47\%) underwent laparoscopic surgery. There was no statistically significant difference between groups in terms of surgical intervention structure $(\mathrm{p}=0.196)$. According to visual estimation, TME performance in both groups was satisfactory. Positional level of tumor location related to anus $(r=0.431$ in $M G$ and $\mathrm{r}=0.417$ in $C G)$ positively correlated $(\mathrm{p}<0.05)$ to distal border of rectum resection $(r=0.510$ and $r=0.532)$, respectively, and inversely correlates to malignancy stage after NCRT $(r=-0.390$ and $r=-0.370)$ involving circumferential resection margin $(C R M+)(r=-0.514$ and $r=-0.522)$. Quality of mesorectumectomy was evaluated after Quirke P et al. However, there was no significant difference between groups in terms of differences in surgical intervention method.

In postsurgical period, surgical material was investigated in terms of morphology. Therapeutic pathomorphosis level was evaluated according to parenchymatous tumor tissue percentage, fibrosis and necrosis levels, considering NCRT conducted (CAPOX regimen and capecitabine-based NCRT). Therapeutic pathomorphosislevel in MG was $42.0 \%$, 58.0 \% 31.2 \%, 51.6 \%; in CG: 56.9 \%, $43.1 \%$, $34.3 \%$, $39.2 \%$, respectively. Evaluation of prescribed NCRT regimen did not confirm higher efficacy of them in terms of stronger pathological response. In this context we talk about polychemotherapy according to CAPOX scheme versus capecitabine-based monochemotherapy. The fact that we did not observe any significant statistical difference in cumulative frequency of PFS between MG and CG (73.5 \pm $8.5 \%$ versus $70.2 \pm 2.5 \%, \mathrm{p}=0.522)$ and correlative general survival $(87 \pm 7.0 \%$ and $96 \pm 2.7 \%)$ proves this. However, according to morphometrical data, there is a statistically significant difference in locoregional therapy response, namely a decrease of parenchymatous tissue and stroma volume $56.9 \%$ and $43.1 \%$ in CG resulting from CAPOX scheme NCRT compared to $42 \%$ i $58 \%$ in MG ( $<<0.001)$. This findings lead to the conclusion that none of above described chemotherapy agents has advantages over the 


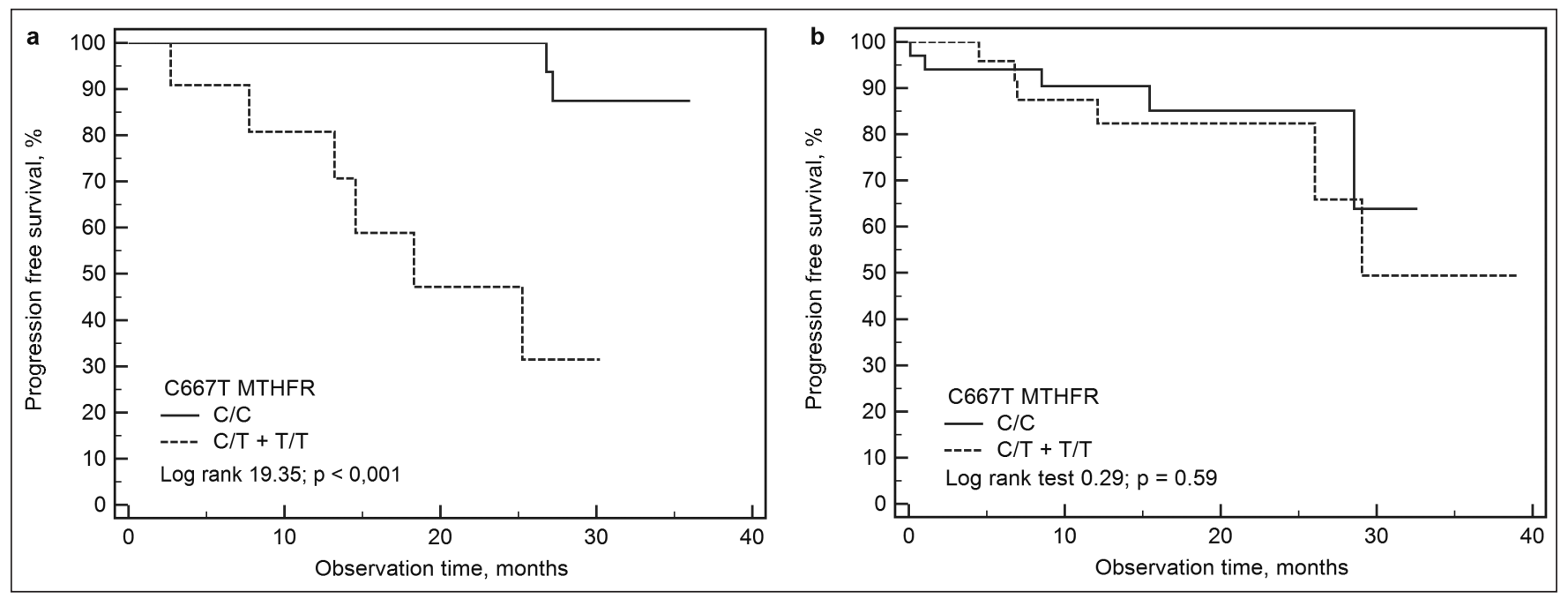

Fig. 2. The effect of C667T MTHFR gene polymorphism on 3-year PFS rate in LARC patients after combined treatment. a - patients received capecitabine as neoadjuvant chemotherapy; $b$ - patients received CAPOX as neoadjuvant chemotherapy.
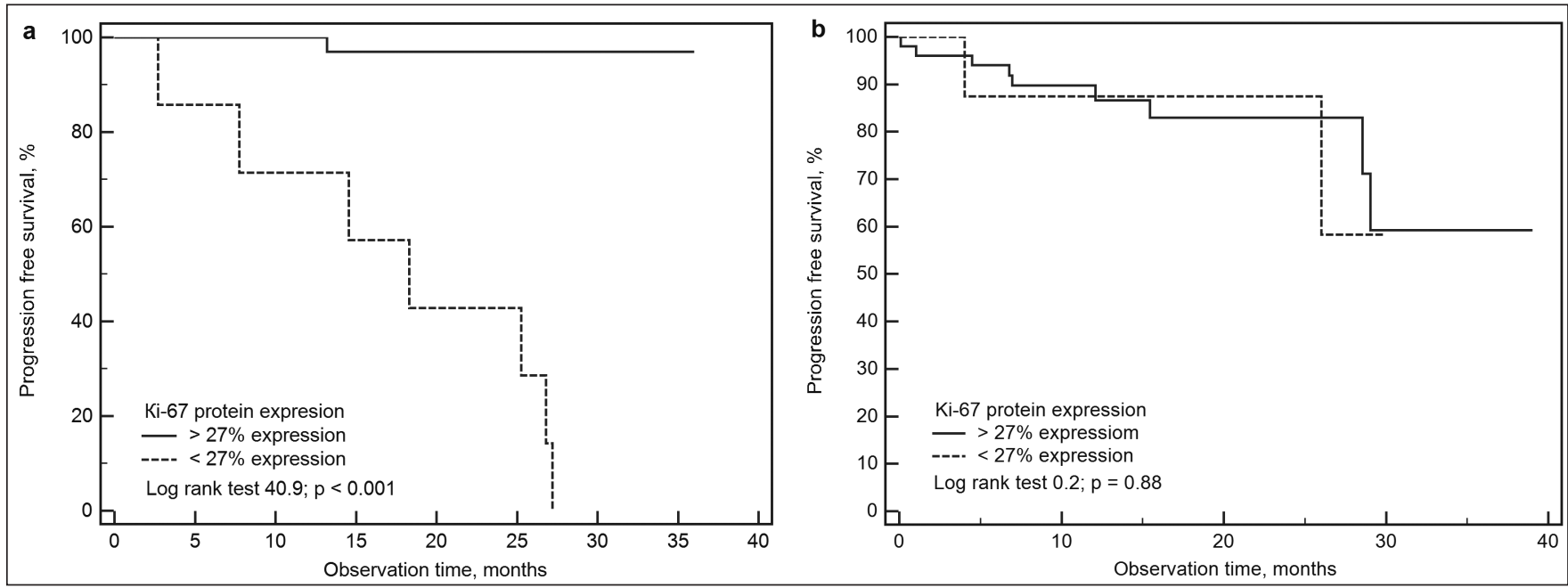

Fig. 3. The effect of Ki-67 protein expression on 3-year PFS rate in LARC patients after combined treatment. a - patients received capecitabine as neoadjuvant chemotherapy; $b$ - patients received CAPOX as neoadjuvant chemotherapy.

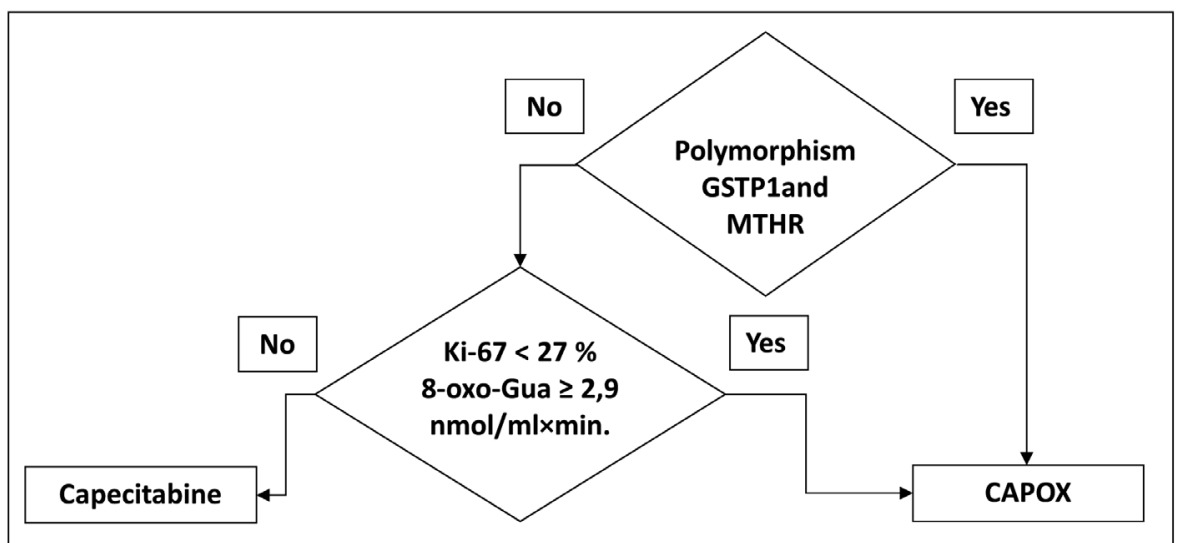

Fig. 4. The personalized algorithm of NCRT application in patients with LARC.

others. A series of international randomized studies listed in introduction came to the same output.

At the same time, to define prognostic factors of rectal cancer course we measured Ki-67 expression levels. The single-factor logistic regression analysis showed that probability of tumor relapse increases when Ki-67 expression level drops. The critical value of Ki-67 expression makes $<27 \%$ where the probability of tumor relapse chance makes 50\%. According to the literature, $\mathrm{Ki}-67$ expression level is a prognostic immunohistochemical biomarker that can predict survival rates decrease in patients with rectal cancer [57]. Comparison of Ki-67 expression level and PFS revealed statistically significant difference in terms of 
Table 1. Recurrence prognostic model in patients with local advanced $\mathrm{RC}$ ( $\beta$ coefficient value and probability levels for independent prognostic factors)

\begin{tabular}{cccc}
\hline Value & $\boldsymbol{\beta}^{*}$ & Vald. $^{* *}$ & $\mathbf{P}$ \\
\hline GSTP 1 & 3,036 & 4,293 & 0,038 \\
\hline MTHFR & 3,169 & 4,270 & 0,039 \\
\hline Ki-67 & $-0,141$ & 4,520 & 0,034 \\
\hline Constant & 1,397 & 0,330 & 0,565 \\
\hline
\end{tabular}

\begin{tabular}{cccc}
\hline GSTP1 & MTHFR & $\begin{array}{c}\text { Ki-67 } \\
<\mathbf{2 7 \%}\end{array}$ & $\begin{array}{c}\text { Recurrence prognostic } \\
\text { probability, (\%) }\end{array}$ \\
\hline+ & + & + & $=99$ \\
\hline+ & + & - & $\geq 62$ \\
\hline+ & - & - & $\geq 73$ \\
\hline- & + & + & $\leq 75$ \\
\hline- & + & - & $\leq 51$ \\
\hline- & - & + & $\geq 51$
\end{tabular}

${ }^{*}-\beta$ coefficient in logistic regression; ${ }^{* *}$ - significance criterion for $\beta$ coefficient.

PFS rate in CG. Patients with Ki-67 expression > 27\% have 27.5 times higher relapse risk compared to patients with Ki-67 expression $<27 \%$. In MG, such statistically significant difference was not observed (Fig.1).

Detection of polymorphism in GSTP1 and MTHFR genes in LARC patients is a new innovative research direction. Threeyear PFS in patients from CG that carry GSTP1 and MTHFR polymorphism is significantly higher. According to logistic regression analysis, relapse risk in patients who carries GSTP1 and MTHFR polymorphism is 12.3 and 16.3 times higher compared to patients without it, respectively. In CG three-year PFS decreases significantly in patients with A313G GSTP1 and C667T MTHFR polymorphisms compared to the patients without these mutations ( $\mathrm{p}<0.001$ ). In $\mathrm{MG}$, there was no statistically significant difference in relapse-free survival level in patients with or without GSTP1 and MTHFR polymorphism (Fig.2, Fig.3).

Based on such factors as GSTP1 and MTHFR gene polymorphism and Ki-67 expression level, we developed a mathematical model of relapse in LARC patients (table I). Based on multifactorial logistic regression analysis next formula was generated (Formula 1):

$$
\mathrm{p}=\frac{1}{1+\mathrm{e}^{-\mathrm{z}}}
$$

where $Z=c+\beta_{\mathrm{f} 1} \times$ GSTP1 $+\beta_{\mathrm{f} 2} \times$ MTHFR $-\beta_{\mathrm{f} 3} \times \mathrm{Ki}-67$; $\mathrm{e}=2.72 ; \mathrm{Z}$ - likelihood of relapse; $\mathrm{c}=1.397$ (constant); $\beta_{\mathrm{f} 1}-$ coefficient for GSTP1 factor; $\beta_{\mathrm{f} 2}-$ coefficient for MTHFR factor; $\beta_{\mathrm{f} 3}-$ coefficient for Ki-67 factor. It helps estimate relapse risk patient-specifically within 3 years after treatment. Prognostic probability of relapse can be evaluated within 51-99\% depending on GSTP1, MTHFR and Ki-67 factors combination.

As a result, personalized algorithm of NCRT selection was developed (Fig.4). In case of GSTP1 and MTHFR polymorphism or their combination as significant prognosis factors, CAPOX scheme NCRT is recommended. If polymorphisms in these genes are absent, Ki-67 $(<27 \%)$ and 8 -oxoGu ( $\geq 2,9 \mathrm{nmol} / \mathrm{mLxmin})$ expression levels should be considered prior to NCRT prescription.

\section{CONCLUSIONS}

The analysis of many scientific publications proves that LARC diagnostics and treatment is an essential and very complicated issue in modern clinical oncology. Based on our own knowledge and practical experience in optimization of combined modality LARC treatment considering prognostic factors (8-oxoGu, Ki-67, A313G GSTP1 and C667T MTHFR) we came to clear understanding of personalized NCRT schemes. The algorithm developed from multifactorial system allows to predict and monitoring LARC relapse development. Consequently, it will improve immediate and postponed results of treatment in patients with this complicated heterogenetic oncological disease.

\section{REFERENCES}

1. National Comprehensive Cancer Network. NCCN Guidelines for Rectal Cancer Version 3. 2017. Available from: URL: https://www.nccn.org/ about/news/ebulletin/ebulletindetail.aspx?ebulletinid=1319

2. Luzietti E, Pellino G, Nikolaou $S$ et al. Comparison of guidelines for the management of rectal cancer. BJS Open. 2018;2(6):433-451.

3. Bhangu A, Beynon J, Brown $G$ et al. Consensus statement on the multidisciplinary management of patients with recurrent and primary rectal cancer beyond total mesorectal excision planes. Br J Surg. 2013;100(8):1009-1014.

4. Arnold M, Sierra MS, Laversanne M et al. Global patterns and trends in colorectal cancer incidence and mortality. Gut. 2017;66(4):683-691.

5. Amann M, Burghardt J, Stratz C et al. Transanal endoscopic microsurgery in treatment of small rectal T1 highrisk, T2 and T3 carcinomas combined with radiochemotherapy. Eur Surg. 2015;47(5):226-237.

6. Verseveld M, de Graaf EJ, Verhoef C et al. CARTS Study Group. Chemoradiation therapy for rectal cancer in the distal rectum followed by organ-sparing transanal endoscopic microsurgery (CARTS study). $\mathrm{Br}$ J Surg 2015;102(7):853-860.

7. Sauer R, Liersch T, Merkel $S$ et al. Preoperative versus postoperative chemoradiotherapy for locally advanced rectal cancer:results of the German CAO/ARO/ AI0-94 randomized phase III trial after a median follow-up of 11 years. J Clin Oncol. 2012;30:1926-1933.

8. Schmoll H, Haustermans K, Price TJ et al. Preoperative chemoradiotherapy and postoperative chemotherapy with capecitabine and oxaliplatin versus capecitabine alone in locally advanced rectal cancer:Disease-free survival results at interim analysis. J Clin Oncol. 2014;32(15):3501-3501.

9. Gérard JP, Azria D, Gourgou-Bourgade $S$ et al. Clinical outcome of the ACCORD 12/0405 PRODIGE 2 randomized trial in rectal cancer. J Clin Oncol. 2012;36:4558-4565.

10. van Gijn W, Marijnen C, Nagtegaal ID et al. Preoperative radiotherapy combined with total mesorectal excision for resectable rectal cancer: 12-year follow-up of the multicentre, randomised controlled TME trial. Lancet Oncol. 2011;12(6):575-582. 
11. Aschele C, Cionini L, Lonardi S et al. Primary tumor response to preoperative chemoradiation with or without oxaliplatin in locally advanced rectal cancer:pathologic results of the STAR-01 randomized phase III trial. J Clin Oncol. 2011;29:2773-2780.

12. Gérard JP, Azria D, Gourgou-Bourgade $S$ et al. Clinical outcome of the ACCORD 12/0405 PRODIGE 2 randomized trial in rectal cancer. J Clin Oncol. 2012;36:4558-4565.

13. Schmoll H, Haustermans K, Price TJ et al. Preoperative chemoradiotherapy and postoperative chemotherapy with capecitabine +/- oxaliplatin in locally advanced rectal cancer:Final results of PETACC-6.JClin Oncol.2018;36(15):3500.

14. Rödel C, Liersch T, Becker H el al. Preoperative chemoradiotherapy and postoperative chemotherapy with fluorouracil and oxaliplatin versus fluorouracil alone in locally advanced rectal cancer:initial results of the German CA0/ARO/AI0-04 randomised phase 3 trial. Lancet Oncol. 2012;13:679-687.

15. RödelC, Graeven U, Fietkau Ret al. Oxaliplatin added to fluorouracilbased preoperative chemoradiotherapy and postoperative chemotherapy of locally advanced rectal cancer (the German CAO/ARO/AI0-04 study):final results of the multicentre, open-label, randomised, phase 3 trial. Lancet Oncol. 2015;16:979-989.

16. Gerdes J, Lemke H, Baisch H et al. Cell cycle analysis of a cell proliferationassociated human nuclear antigen defined by the monoclonal antibody Ki-67. J Immunol. 1984;133(4):1710-1715.

17. Gerdes J, Stein H, Pileri S et al. Prognostic relevance of tumour-cell growth fraction in malignant non-Hodgkin's lymphomas. Lancet. 1987;330(8556):448-449.

18. Dowsett M, Nielsen T0, A'Hern Ret al. International Ki-67 in Breast Cancer Working Group. Assessment of Ki67 in breast cancer:recommendations from the International Ki67 in Breast Cancer working group. J Natl Cancer Inst. 2011;103(22):1656-1664.

19. Luo Y, Ren F, Liu Y et al. Clinicopathological and prognostic significance of high Ki-67 labeling index in hepatocellular carcinoma patients:a meta-analysis. Int J Clin Exp Med. 2015;8(7):10235-10247.

20. Pezzilli R, Partelli S, Cannizzaro R et al. Ki-67 prognostic and therapeutic decision driven marker for pancreatic neuroendocrine neoplasms (PNENs):A systematic review. Adv Med Sci. 2016;61(1):147-153.

21. Richards-Taylor S, Ewings SM, Jaynes E et al. ReviewThe assessment of Ki-67 as a prognostic marker in neuroendocrine tumours:a systematic review and meta-analysis. J Clin Pathol. 2016;69(7):612-618.

22. Pap $Z$, Ilyes IA, Mocan SL et al. Changes in immunoexpression of $\mathrm{p} 53$, Ki-67, Ets-1, APAF-1 and PTEN in serrated and conventional colon adenomas. Rom J Morphol Embryol. 2015;56(4):1389-1396.

23. Hayashi H, Beppu T, Sakamoto Y et al. Prognostic value of Ki-67 expression in conversion therapy for colorectal liver-limited metastases. Am J Cancer Res. 2015;5(3):1225-1233.

24. Fernandez-Cebrian JM, Nevado SM, Vorwald KP et al. Can the clinical outcome in stage II colon carcinomas be predicted by determination of molecular marker expression? Clin Transl Oncol. 2007;9(10):663-670.

25. WuXS, XiHQ, Chen L. Lgr 5 is a potential marker of colorectal carcinoma stem cells that correlates with patient survival. World J Surg Oncol. 2012;10:244.

26. Furudoi A, Tanaka S, Haruma $K$ et al. Clinical significance of human erythrocyte glucose transporter 1 expression at the deepest invasive site of advanced colorectal carcinoma. Oncology. 2001;60(2):162-169.

27. Lin MX, Wen ZF, Feng ZY, He D. Expression and significance of Bmi-1 and Ki67 in colorectal carcinoma tissues. Ai Zheng. 2008;27(12):1321-1132.

28. Salminen E, Palmu S, Vahlberg T et al. Increased proliferation activity measured by immunoreactive Ki67 is associated with survival improvement in rectal/recto sigmoid cancer. World J Gastroenterol. 2005;11(21):3245-3245.
29. Xi HQ, Zhao P. Clinicopathological significance and prognostic value of EphA3 and CD133 expression in colorectal carcinoma. J Clin Pathol. 2011;64(6):498-503.

30. Ivanecz A, Kavalar R, Palfy M et al. Can we improve the clinical risk score? The prognostic value of $\mathrm{p} 53, \mathrm{Ki}-67$ and thymidylate synthase in patients undergoing radical resection of colorectal liver metastases. HPB (0xford). 2014;16(3):235-242.

31. Libutti SK, Saltz LB, Tepper JE. Cancer of the Colon. In: DeVita VT. Lawrence TS, Rosenberg SA et al. Cancer: Principles \& Practice of Oncology. Philadelphia: Lippincott Williams \& Wilkins; 2011, p. 1084-1153.

32. Polat ND, Degirmencioglu S, Yaren A et al. GSTP1, TSER, MTHFR C677T and MTHFR A1298C gene single nucleotide polymorphisms associated with toxicity and survival in patients with colorectal cancer treated with 5-fluorouracil-based chemotherapy. Cancer Rep Rev. 2018;2(2):1-7.

33. Mohile S, Dale W, Somerfield M et al. Practical Assessment and Management of Vulnerabilities in Older Patients Receiving Chemotherapy:ASCO Guideline for Geriatric Oncology. J Oncol Pract. 2018; 14(7):442-446.

34. Chéradame $S$, Etienne MC, Chazal M et al. Relevance of tumoral folylpolyglutamate synthetase and reduced folates for optimal 5-fluorouracil efficacy:experimental data. Eur J Cancer. 1997;33:950-959.

35. Sharma R, Hoskins JM, Rivory LP et al. Thymidylate Synthase and Methylenetetrahydrofolate Reductase Gene Polymorphisms and Toxicity to Capecitabine in Advanced Colorectal Cancer Patients. Clin Cancer Res. 2008;14(3):817-825.

36. Etienne MC, Formento JL, Chazal M et al. Methylenetetrahydrofolate reductase gene polymorphisms and response to fluorouracil-based treatment in advanced colorectal cancer patients. Pharmacogenetics, 2004;14:785-792.

37. Ali-Osman F, Akande 0, Antoun G et al. Molecular cloning, characterization, and expression in Escherichia coli of full-length cDNAs of three human glutathione S-transferase Pi gene variants. Evidence for differential catalytic activity of the encoded proteins. J Biol Chem. 1997;272(15):10004-10012.

38. Ming-Ju Tsai, Wei-An, Chang Ming-Shyan Huang et al. Tumor microenvironment: A new treatment target for cancer. ISRN Biochemistry, 2014;2014:351959.

39. Said AH, Raufman JP, Xie G. The role of matrix metalloproteinases in colorectal cancer. Cancers Basel. 2014;6(1):366-375.

40. Kasai H. Analysis of a form of oxidative DNA damage, 8-hydroxy2'-deoxyguanosine, as a marker of cellular oxidative stress during carcinogenesis. Mutation Research. 1997;387:147-163.

41. Burlaka A.A., Vovk A.V., Zvirych V.V. et al. Zhyrova tkanyna ta yiyi rol' u mikrootochenni klityn adenokartsynomy kolorektal'noho raku [Role of the adipose tissue in the rectal cancer microenvironment]. Klinichna Onkolohiya. 2019;9(1):30-33. (in Ukraine).

42. Lee HT, Lin CS, Lee CS et al. Increased 8-hydroxy-2'-deoxyguanosine in plasma and decreased mRNA expression of human 8-oxoguanine DNA glycosylase 1, anti-oxidant enzymes, mitochondrial biogenesis-related proteins and glycolytic enzymes in leucocytes in patients with systemic lupus erythematosus. Clin Exp Immunol. 2014;176(1):66-77.

43. Nakabeppu Y, Sakumi K, Sakamoto K, et al. Mutagenesis and carcinogenesis caused by the oxidation of nucleic acids. Biol Chem. 2006;387:373-379.

44. Evans MD, Mistry V, Singh R et al. Nucleotide excision repair of oxidised genomic DNA is not a source of urinary 8-0x0-7,8-dihydro-2'deoxyguanosine. Free Radic Bio Med. 2016;99,385-391. 
45. Taylor FG, Quirke P, Heald RJ et al. Magnetic Resonance Imaging in RectalCancer European Equivalence Study Study Group. Preoperative magnetic resonanceimaging assessment of circumferential resection margin predicts disease-freesurvival and local recurrence:5-year followup results of the MERCURY study. J Clin Oncol. 2014;32(1):34-43.

46. Hyun JW, Jung YC, Kim HS et al. 8-Hydroxydeoxyguanosine causes death of human leukemia cells deficient in 8-oxoguanine glycosylase 1 activity by inducing apoptosis. Mol Cancer Res. 2003;1(4),290-299.

47. Sliwinska A, Kwiatkowski D, Czarny $P$ et al. The levels of 7,8-dihydrodeoxyguanosine (8-0xoG) and 8-oxoguanine DNA glycosylase 1 (OGG1) - A potential diagnostic biomarkers of Alzheimer's disease. J Neurol Scienc. 2016;368:155-159.

This study was supported by the Basic Science Research Program of the Ministry of Health of Ukraine. The dissertation work was carried out within the framework of two planned budgetary works of the National Cancer Institute: "To develop methods of surgical and adjuvant treatment for patients with abdominal organs malignant tumors" (state registration number 0115U000810; topic code VN.14.01.07.14012; deadline 2015-2017), "Development new methods of combined treatment for patients with metastatic neoplasms of the abdominal cavity, retroperitoneal space and pelvis" (state registration number 0118U003734; topic code VN.14.01.07.179-18; deadline 20 18-2020).

\section{ORCID and contributionship:}

Vitalii Zvirych - 0000-0002-3502-1886 ${ }^{\text {B,D }}$

Yuriy Michailovich - 0000-0003-3551-2148 A, D, F, E

Oleksandr Gorbach - 0000-0003-2922-6049 B, C, D

Natalia Khranovska - 0000-0002-0800-2540 B,E

\section{Conflict of interest:}

The authors have no financial conflicts of interest.

\section{CORRESPONDING AUTHOR Vitalii V.Zvirych \\ National Cancer Institute \\ Lomonosov Str, 33/43, 03022, Kyiv, Ukraine \\ tel: +380988508076 \\ e-mail: zvirvit@ukr.net}

Received: 17.01.2020

Accepted: 05.03 .2020

A - Work concept and design, B - Data collection and analysis, C - Responsibility for statistical analysis, $\mathbf{D}$-Writing the article, $\mathbf{E}$-Critical review, $\mathbf{F}$ - Final approval of the article 